Pacific Journal of Mathematics

AND EEMISMPFi cLasses
Rings 


\section{AXIOMATIC RADICAL AND SEMISIMPLE CLASSES OF RINGS}

JOHN R. FISHER

The results of this paper combine two areas of abstract mathematics: the theory of radicals for associative or alternative rings, and model theory for universal algebras. The main theorem provides necessary and sufficient conditions on the radical of any product ring and on the radical of any ultraproduct ring in order that the radical class and its corresponding semisimple class be finitely axiomatic (elementary). As a corollary, it follows that if a radical class of rings and its corresponding semisimple class are axiomatic, then they are both finitely axiomatic. In addition, several subsidiary results are given, and unanswered questions posed.

1. Introduction. Let $\mathscr{A}$ denote either the class of all associative rings, or the class of all alternative rings. Suppose that $\mathscr{R}$ is a subclass of $\mathscr{A}$. For any ring $A$ in $\mathscr{A}$, let $\mathscr{R}(A)$ denote the sum of all the ideals of $A$ which belong to $\mathscr{R}$. Let $S(\mathscr{R})$ denote the class of all rings in $\mathscr{A}$ having no nonzero ideals belonging to $\mathscr{R}$. Then $\mathscr{R}$ is a radical class in $\mathscr{A}$ provided the following properties (R1), (R2), and (R3) hold.

(R1) $\mathscr{R}$ is closed under homomorphic images.

(R2) For every ring $A \in \mathscr{A}, \mathscr{R}(A) \in \mathscr{R}$.

(R3) For every ring $A \in \mathscr{A}$, the quotient ring $A / \mathscr{R}(A) \in S(\mathscr{R})$. The rings in $S(\mathscr{R})$ are called semisimple with respect to $\mathscr{R}$. Properties (R4) through (R7) below are useful consequences of properties (R1), (R2), and (R3). The ring $A$ is assumed to belong to the class $\mathscr{A}$.

(R4) If $I$ is an ideal of $A$ such that $I \in \mathscr{R}$ and $A / I \in \mathscr{R}$, then $A \in \mathscr{R}$. This is called the extension property of radicals.

(R5) If $I$ is an ideal of $A$ and if $A / I \in S(\mathscr{R})$, then $\mathscr{R}(A) \leqq I$.

(R6) $\mathscr{R} \cap S(\mathscr{R})$ consists of the trivial ring (0).

(R7) $S(\mathscr{R})$ is hereditary; that is, if $A \in S(\mathscr{R})$ and if $I$ is an ideal of $A$, then $I \in S(\mathscr{R})$.

References [4], [8], and [13] will provide the reader with the fundamentals of radical theory for rings. Properties (R1) through (R6) are basic facts. Property (R7) is a result from [2], and can be found also in [13, Corollary 5.3 or Theorem 8.1] for associative rings. The radical class $\mathscr{R}$ in $\mathscr{A}$ is called hereditary provided property (R8) holds.

(R8) If $A \in \mathscr{R}$ and $I$ is an ideal of $A$, then $I \in \mathscr{R}$. 
For a technical definition of an axiomatic class in model theory, the reader is referred to [3] or [7]. Roughly stated, the subclass $\mathscr{R}$ of $\mathscr{A}$ is axiomatic, respectively finitely axiomatic (or elementary), provided there is a set, respectively a finite set, $S$ of formal ring sentences (made up from variables, ring operations, equality, and quantifiers) such that $\mathscr{R}$ is the class of all rings in $\mathscr{A}$ that satisfy all the sentences of $S$. A relevant example here is the class of all Jacobson radical rings, which can be described as the class of all associative rings satisfying the formal ring sentence $\forall x \exists y(x+y+x y=0)$.

The last definition needed before stating the Main Theorem is that of an ultraproduct of rings. References [3] and [7] will again serve as technical sources. For rings in $\mathscr{A}$, the definition is given below. Suppose that $I$ is a set. A filter on $I$ is a collection $F$ of subsets of $I$ such that (a) if $H, J \in F$ then $H \cap J \in F$; (b) if $H \in F$ and $J$ is a subset of $I$ such that $H \subseteq J$ then $J \in F$; (c) the empty set does not belong to $F$. Given a family $\left\{A_{i} \mid i \in I\right\}$ of rings in $\mathscr{R}$, let $A=\pi A_{i}$ be the product ring of the family, and let

$$
K=\{a \in A \mid\{i \mid a(i)=0\} \in F\} .
$$

One can check directly that $K$ is an ideal of $A$. The quotient ring $A_{F}=A / K$ is the reduced product of the family modulo the filter $F$ on $I$. If $F$ is a maximal filter on $I$ (one not properly contained in any other filter on $I$ ), then $A_{F}$ is called the ultraproduct of the family modulo the ultrafilter $F$. If $\mathscr{R}$ is a radical class of rings in $\mathscr{A}$, one can consider the ideal $L$ of $A$, defined as follows:

$$
L=\left\{a \in A \mid\left\{i \mid a(i) \in \mathscr{R}\left(A_{i}\right)\right\} \in F\right\} .
$$

Note that $K \cong L$.

MAIN THeOREM. Suppose that $\mathscr{R}$ is a radical class of rings. Consider the following conditions on $\mathscr{R}$ :

(A) $\mathscr{R}$ is closed under arbitrary products of members of $\mathscr{R}$.

(B) If $I$ is a set, $F$ an ultrafilter on $I$, and $\left\{A_{i} \mid i \in I\right\}$ is a family of rings, then

$$
\mathscr{R}\left(A_{F}\right) \subseteq L / K
$$

where

$$
K=\left\{a \in \pi A_{i} \mid\{i \mid a(i)=0\} \in F\right\},
$$

and

$$
L=\left\{a \in \pi A_{i} \mid\left\{i \mid a(i) \in \mathscr{R}\left(A_{i}\right)\right\} \in F\right\} .
$$


The following implications hold:

(1) If $\mathscr{R}$ and $S(\mathscr{R})$ are both axiomatic, then $\mathscr{R}$ satisfies conditions (A) and (B).

(2) If $\mathscr{R}$ satisfies conditions (A) and (B), then both $\mathscr{R}$ and $S(\mathscr{R})$ are finitely axiomatic.

The proof of the Main Theorem is in §II. The proof depends heavily on the results of [3, $\S \mathrm{V} .6$ and VI. 6], using versions of the theorems there relativized to the elementary model class . of rings, and requires the generalized continuum hypothesis. The following corollary is an easy consequence of the Main Theorem.

Corollary. If $\mathscr{R}$ is a radical class of rings such that $\mathscr{R}$ and $S(\mathscr{R})$ are both axiomatic, then $\mathscr{R}$ and $S(\mathscr{R})$ are both finitely axiomatic.

In $\S$ III it will be shown that the ideal $L / K$ of the reduced product $A_{F}$ is naturally isomorphic to the reduced product

$$
\left[\pi \mathscr{R}\left(A_{2}\right)\right]_{F}=\mathscr{R}(A)_{F},
$$

provided $\mathscr{R}$ is closed under products. If $\mathscr{R}$ is closed under products and if $\mathscr{R}(A)_{F}$ is identified with $L / K$, then condition (B) of the Main Theorem could be written $\mathscr{R}\left(A_{F}\right) \subseteq \mathscr{R}(A)_{F}$.

The axiomatic radical classes of associative rings known to the author are all finitely axiomatic. These radicals are described below. ties.

(i) Radical classes determined by certain polynomial regulari-

See $\S$ III for a more complete discussion and references. Well-known examples include the Jacobson radical, with axiom $\forall x \exists y(x+y+$ $x y=0)$, and the von Neumann regular radical, with axiom $\forall x \exists y(x y x-x=0)$.

(ii) The radical-semisimple classes $V(P, N)$ characterized in [6].

These radical classes are subvarieties of rings. Included in this characterization are the radical-semisimple classes $\mathscr{K}_{n}(n=1,2, \cdots)$, where $\mathscr{K}_{n}$ has defining axiom $\forall x\left(x^{n}=0\right)$.

Proposition 2 of $\S$ III gives sufficient conditions for $S(\mathscr{R})$ to be axiomatic, and displays a defining axiom for $S(\mathscr{R})$. The Jacobson radical satisfies the conditions of Proposition 2, but the question of which other axiomatic radical classes have an axiomatic semisimple class remains open at this writing.

Proposition 3 of $\S$ III gives sufficient conditions, again satisfied by the Jacobson radical, in order for the containment $\mathscr{R}\left(A_{F}\right) \subseteq$ 
$\mathscr{R}(A)_{F}$ of $(\mathrm{B})$ of the Main Theorem to be an equality for all ultraproducts $A_{F}$ of rings.

Ultraproducts of associative rings have been used with success in connection with the theory of prime rings satisfying polynomial identities; see [1], [9, $\S 3$ of Chapter 7], or [11].

\section{Proof of the Main Theorem.}

LemMa. Suppose that $\mathscr{R}$ is a radical class of rings.

(a) If $I$ is a set and $\left\{A_{i} \mid i \in I\right\}$ is a family of rings, then

$$
\mathscr{R}\left(\pi A_{i}\right) \leqq \pi \mathscr{R}\left(A_{i}\right) .
$$

(b) $\mathscr{R}$ is closed under arbitrary products if and only if

$$
\mathscr{R}\left(\pi A_{i}\right)=\pi \mathscr{R}\left(A_{i}\right)
$$

for any set $I$ and family of rings $\left\{A_{i} \mid i \in I\right\}$.

(c) If $A_{1}, \cdots, A_{n}$ are rings, then

$$
\mathscr{R}\left(A_{1} \times \cdots \times A_{n}\right)=\mathscr{R}\left(A_{1}\right) \times \cdots \times \mathscr{R}\left(A_{n}\right) .
$$

Hence, $\mathscr{R}$ is always closed under finite products.

(d) $\mathscr{R}$ is closed under products if and only if $\mathscr{R}$ is closed under reduced products.

(e) If $\mathscr{R}$ is axiomatic, then $\mathscr{R}$ is closed under arbitrary products.

Proof. Items (a), (b), and (c) are fairly straightforward and can be found in [10]. In particular, (c) follows by induction after one has proved that the product of two radical rings is radical, and the latter can be accomplished using the extension property (R4) of radicals. To be technically correct, one should note that the product of the empty family of radical rings is the trivial ring (0), and $(0) \in \mathscr{R}$. Item (d) follows, on the one hand, from the fact that any reduced product $\left(\pi A_{i}\right)_{F}=\pi A_{i} / K$ is a homomorphic image of a product, so that if the product $\pi A_{i}$ belongs to $\mathscr{R}$ then the reduced product $\left(\pi A_{i}\right)_{F}$ belongs to $\mathscr{R}$ by (R1). Conversely, any product $\pi A_{i}$ is isomorphic to the reduced product $\left(\pi A_{i}\right)_{F}$, where $F=\{I\}$, hence if $\mathscr{R}$ is closed under all reduced products, then $\mathscr{R}$ is closed under all products. For (e), a theorem in model theory applies [7, p. 292]. This theorem, stated for associative or alternative rings, states that an axiomatic class of rings which is closed under finite products is also closed under arbitrary products.

(1) Suppose that $\mathscr{R}$ and $S(\mathscr{R})$ are both axiomatic. Then (A) 
follows by the Lemma (e). For (B), suppose that $I$ is a set, $F$ an ultrafilter on $I$, and that $\left\{A_{i} \mid i \in I\right\}$ is a family of rings. Let $R_{i}=$ $\mathscr{R}\left(A_{i}\right)$, for all $i \in I$, and $A=\pi A_{i}$. Also let

$$
\begin{aligned}
& K=\{a \in A \mid\{i \mid a(i)=0\} \in F\}, \\
& L=\left\{a \in A \mid\left\{i \mid a(i) \in R_{i}\right\} \in F\right\},
\end{aligned}
$$

and

$$
\mathscr{K}=\left\{f \in \pi\left(A_{i} / R_{i}\right) \mid\{i \mid f(i)=0\} \in F\right\} .
$$

Then a straightforward check will confirm that

$$
\dot{\phi}: A / L \longrightarrow \pi\left(A_{i} / R_{i}\right) / \mathscr{K}
$$

is an isomorphism, where

$$
\phi(a+L)=\hat{a}+K,
$$

and where $\hat{a} \in \pi\left(A_{i} / R_{i}\right)$ is such that, for $i \in I$,

$$
\hat{a}(i)=a(i)+R_{i} .
$$

Since $S(\mathscr{R})$ is axiomatic, $S(\mathscr{R})$ is closed under ultraproducts [3, Corollary 6.6, p. 244]. Hence, the ultraproduct $\pi\left(A_{i} / R_{i}\right) / \mathscr{K}$ belongs to $S(\mathscr{R})$, since each $A_{i} / R_{i} \in S(\mathscr{R})$ by (R3). Thus, $A / L \in S(\mathscr{R})$. But $A / L \cong(A / K) /(L / K)$, and so $\mathscr{R}(A / K)=\mathscr{R}\left(A_{F}\right) \subseteq L / K$ by (R5). Consequently, (B) also holds.

(2) Suppose that conditions (A) and (B) hold. To prove that $\mathscr{R}$ is finitely axiomatic, it suffices [3, Corollary 6.6, p. 244, relativized to the model class $\mathscr{A}$ of rings] to show that both $\mathscr{R}$ and $\mathscr{R}^{\prime}$, the complement of $\mathscr{R}$ in the model class $\mathscr{A}$ of rings, are closed under ultraproducts. $\mathscr{R}$ is closed under ultraproducts by the Lemma (d). To show that $\mathscr{R}^{\prime}$ is closed under ultraproducts, suppose that the ultraproduct $A_{F}=\pi A_{i} / K$ belongs to $\mathscr{R}$. Then $\mathscr{R}\left(A_{F}\right)=\mathscr{R}\left(\pi A_{i} / K\right)=\pi A_{i} / K$, and so, by $(\mathrm{B}), \pi A_{i} / K=L / K$. From this it follows that some $A_{i}$ belongs to $\mathscr{R}$. For suppose that, for each $i, R_{i}=\mathscr{R}\left(A_{i}\right) \neq A_{i}$. Then, for each $i$, there exists $a_{i} \in A_{i} \backslash R_{i}$. Let $a \in \pi A_{i}$ be such that $a(i)=a_{i}$ for each $i$. But then $a+K=$ $b+K$ for some $b \in L$. Let $S_{1}=\{i \mid a(i)=b(i)\}, \quad S_{2}=\left\{i \mid b(i) \in R_{i}\right\}$. Both $S_{1}$ and $S_{2}$ belong to $F$. Since $F$ is a filter, $S_{1} \cap S_{2}$ is not empty. Thus there exists some $j \in S_{1} \cap S_{2}$. Consequently, $a_{j}=a(j)=$ $b(j) \in R_{j}$, contrary to $a_{j} \notin R_{j}$. Hence some $A_{i}$ must belong to $\mathscr{R}$. Thus $\mathscr{R}^{\prime}$ is closed under ultraproducts, and so $\mathscr{R}$ is finitely axiomatic.

It remains to show that $S(\mathscr{R})$ is finitely axiomatic. Suppose that $\left\{A_{i} \mid i \in I\right\}$ is a family of rings in $S(\mathscr{R})$, and that $F$ is an ultrafilter on $I$. Set $A=\pi A_{i}$. Then $\mathscr{R}\left(A_{F}\right) \subseteq L / K$, by (B). But 
since each $A_{i} \in S(\mathscr{R}), L=K$, so $\mathscr{R}\left(A_{F}\right)=(0)$. Thus $A_{F} \in S(\mathscr{R})$, and so $S(\mathscr{R})$ is closed under ultraproducts. To show that $S(\mathscr{R})^{\prime}$ is closed under ultraproducts, suppose that the ultraproduct $A_{F}=\pi A_{i} / K$ belongs to $S(\mathscr{R})$. Let $R_{i}=\mathscr{R}\left(A_{i}\right)$ for each $i$. Then $R=\pi R_{i}$ belongs to $\mathscr{R}$, by (A). Moreover, $(R+K) / K \cong R /(R \cap K)$, and $R /(R \cap K)$ belongs to $\mathscr{R}$ by (R1). But, by (R7), $(R+K) / K$ belongs to $S(\mathscr{R})$ since $(R+K) / K$ is an ideal of $A / K$, and $A / K=A_{F} \in S(\mathscr{R})$. Thus $(R+K) / K \in \mathscr{R} \cap S(\mathscr{R})$, and so $(R+K) / K=(0)$ by (R6). Hence $R=\pi R_{i} \subseteq K$. One can easily show that this implies that some $R_{j}=(0)$, and consequently $A_{j} \in S(\mathscr{R})$. Therefore $S(\mathscr{R})^{\prime}$ is also closed under ultraproducts. Thus $S(\mathscr{R})$ is finitely axiomatic.

III. Further results, comments, and questions. Suppose that $\mathscr{R}$ is a radical class of rings that is closed under products. Suppose further that $\left\{A_{i} \mid i \in I\right\}$ is a family of rings, and that $F$ is a filter on the set $I$. Let $R_{i}=\mathscr{R}\left(A_{i}\right)$ for each $i \in I, A=\pi A_{i}$, and $R=\pi R_{i}$. Then $\mathscr{R}(A)=\mathscr{R}\left(\pi A_{i}\right)=\pi R_{i}$ by the Lemma (b) in $\S$ II. Let

$$
K=\{a \in A \mid\{i \mid a(i)=0\} \in F\}
$$

and

$$
K_{R}=\{r \in R \mid\{i \mid r(i)=0\} \in F\}=R \cap K .
$$

Then

$$
R_{F}=R / K_{R}=R /(R \cap K) \cong(R+K) / K=L / K \cong A_{F},
$$

where, as before,

$$
L=\left\{a \in A \mid\left\{i \mid a(i) \in R_{i}\right\} \in F\right\} .
$$

Thus, if $L / K$ is identified with its isomorphic copy $R_{F}$, condition (B) of the Main Theorem would read $\mathscr{R}\left(A_{F}\right) \subseteq \mathscr{R}(A)_{F}$.

Every axiomatic radical class must be closed under products, by the Lemma (e) in $\S$ II. Since the lower Baer radical, the Levitzki radical, and the nil radical are not closed under products, these radical classes for associative rings are not axiomatic. It also appears unlikely that the Brown-McCoy radical class is closed under products; see [10, pp. 56-57].

At the end of $\S I$, it was stated that certain polynomial regularities determine finitely axiomatic radical classes for associative rings. The concept of a polynomial regularity is characterized in reference [12]. A brief statement of the necessary definitions is given below, with a slight change of notation.

Suppose that $p_{1}, \cdots, p_{n}$ are integral polynomials. For each associative ring $A$ define function $\mathscr{R}_{A}$ as follows: 


$$
\mathscr{R}_{A}(a)=p_{1}(a) A p_{2}(a) A \cdots A p_{n}(a),
$$

for every $a \in A . \quad \mathscr{R}_{A}$ is a function from the ring $A$ into the class of all subgroups of the additive group of $A$. That function which maps a ring $A$ to the corresponding $\mathscr{R}_{A}$ is called a polynomial regularity. An element $a \in A$ is said to be $\mathscr{R}_{A}$-regular provided $a \in \mathscr{R}_{A}(a) . A$ is said to be $\mathscr{R}$-regular provided each element of $A$ is $\mathscr{R}_{A}$-regular. The class of all $\mathscr{R}$-regular rings is a radical class of rings, which is conveniently denoted by $\mathscr{R}$. That is,

$$
\mathscr{R}=\left\{A \mid a \in \mathscr{R}_{A}(a) \text { for all } a \in A\right\} .
$$

$\mathscr{R}$ is said to be the radical class determined by the corresponding polynomial regularity.

Proposition 1. Suppose that $\mathscr{R}$ is the radical class of associative rings determined by a polynomial regularity. Then the following statements are equivalent:

(1) $\mathscr{R}$ is axiomatic.

(2) $\mathscr{R}$ is closed under products.

Proof. (1) implies (2) by the Lemma (e) of $\S$ II. Conversely, suppose that $\mathscr{R}$ is closed under products. Then $\mathscr{R}$ is closed under ultraproducts by the Lemma (d) of $\S$ II. To show that $\mathscr{R}$ is axiomatic, it suffices [3, Corollary 6.5, relativized to the model class of associative rings] to show that $\mathscr{R}^{\prime}$, the class of all associative rings not belonging to $\mathscr{R}$, is closed under ultrapowers. This result is implied by the following lemma.

Lemma. Suppose that $\mathscr{R}$ is the radical class of associative rings determined by a polynomial regularity. Then the class $\mathscr{R}^{\prime}$ of all associative rings not belonging to $\mathscr{R}$ is closed under reduced products.

Proof. Suppose that some reduced power $U=A^{I} / K$ of the ring $A$ belongs to $\mathscr{R}$. Let $F$ denote the underlying filter. Suppose further that $\mathscr{R}$ has the following representation:

$$
\mathscr{C}_{A}(a)=p_{1}(a) A p_{2}(a) A \cdots A p_{n}(a)
$$

for any $a \in A$. Given $a \in A$, let $\widehat{a} \in A^{I}$ be such that $\widehat{a}(i)=a$, for all $i \in I$. Since $U \in \mathscr{R}, \hat{a}+K \in \mathscr{R}_{l}(\hat{a}+K)$. However,

$$
\begin{aligned}
\mathscr{R}_{U}(\hat{a}+K) & =p_{1}(\hat{a}+K) U p_{2}(\hat{a}+K) U \cdots U p_{n}(\hat{a}+K) \\
& =\left[p_{1}(\hat{a}) A^{I} p_{2}(\hat{a}) A^{I} \cdots A^{I} p_{n}(\hat{a})+K\right] / K .
\end{aligned}
$$


Thus, there exists a positive integer $k$, and $f_{1 i}, f_{2 i}, \cdots, f_{n-1, i}$ belonging to $A^{I}$, for $i=1, \cdots, k$, such that

$$
\widehat{a}-\sum_{i=1}^{k} p_{1}(\widehat{a}) f_{1 i} p_{2}(\widehat{a}) f_{2 i} \cdots f_{n-1, i} p_{n}(\hat{a}) \in K \text {. }
$$

Hence, the set

$$
S=\left\{j \mid \widehat{a}(j)=\sum_{i=1}^{k} p_{1}(\hat{a}) f_{1 i} p_{2}(\hat{a}) f_{2 i} \cdots f_{n-1, i} p_{n}(\hat{a})(j)\right\}
$$

belongs to $F$. Thus $S$ is not empty. Let $s \in S$. Then

$$
\hat{a}(s)=\sum_{i=1}^{k} p_{1}(\hat{a}) f_{1 i} p_{2}(\hat{a}) f_{2 i} \cdots f_{n-1, i} p_{n}(\hat{a})(s),
$$

or

$$
a=\sum_{i=1}^{k} p_{1}(a) f_{1 i}(s) p_{2}(a) f_{2 i}(s) \cdots f_{n-1, i}(s) p_{n}(a),
$$

that is, $a \in \mathscr{R}_{A}(a)$. Consequently $A \in \mathscr{R}$. Thus, $\mathscr{R}^{\prime}$ is closed under reduced powers, as claimed.

The conjecture that the Brown-McCoy radical class is not closed under products was stated earlier. In view of Proposition 1, this conjecture is equivalent to saying that the Brown-McCoy radical class is not axiomatic. A representation of the Brown-McCoy radical class as a polynomial regularity is $\mathscr{B}_{A}(a)=A(1+a)+A(1+a) A$. The element $a$ of the ring $A$ is $\mathscr{B}_{A}$-regular provided there exists some positive integer $k$, and $b, c_{i}, d_{i} \in A(i=1, \cdots, k)$, such that $a=b(1+a)+\sum_{i=1}^{k} c_{i}(1+a) d_{i}$. To say that $\mathscr{B}$ is axiomatic would seem to force some upper bound on the numbers $k$ for representing all such sums as above, so that $\mathscr{B}$ could have as a possible defining axiom some sentence of the form $\forall x \exists y_{i} \exists y_{2} \cdots \exists y_{k}(\cdots)$. This remains conjecture. More generally, we could ask the following question:

Question 1. If $\mathscr{R}$ is an axiomatic radical class determined by a polynomial regularity, does representation of $\mathscr{R}$ have to take a form $\mathscr{R}_{A}(a)=p_{1}(a) A p_{2}(a)$, using exactly two integral polynomials $p_{1}$ and $p_{2}$ ?

It should be observed that each of the axiomatic radical classes given at the end of $\S$ I can be defined by a single universal-existential axiom. In general, a universal-existential sentence is one that has the form $\forall x_{1} \cdots \forall x_{m} \exists y_{1} \cdots \exists y_{n} P$, where $P$ does not contain quantifiers; included is the case where universal quantifiers are absent or existential quantifiers are absent, as in the case of 
$\forall x\left(x^{3}=0\right)$. A result in model theory [7, Theorem 2, p. 279] gives the following. Suppose that $\mathscr{R}$ is an axiomatic class of rings in $\mathscr{A}$, and that $S$ is the set of all universal-existential ring sentences that hold in $\mathscr{R}$. Then $\mathscr{R}$ is exactly the class of all rings in $\mathscr{A}$ satisfying the sentences in $S$ if and only if $\mathscr{R}$ is closed under countable chain unions. A countable chain union of rings consists of a union ring $R=\cup R_{i}$, where $R_{1} \subseteq R_{2} \subseteq \cdots$ is a countable chain of rings, and where each $R_{i}$ is a subring of $R_{i+1}$. Most of the known radical classes, whether axiomatic or not, are closed under countable chain unions. For example, the nil radical, the Brown-McCoy radical, the Jacobson radical, the von Neumann regular radical, the Levitzki radical, and $\mathscr{K}_{n}$ radicals mentioned in $\S I$ are all closed under countable chain unions. Moreover, any radical class for associative rings determined by a polynomial regularity is closed under countable chain unions. (Thus, if such a radical class were axiomatic, it would have some axiomatization using universal-existential axioms, as suggested in the paragraph preceding Question 1.) Other radical classes may not be closed under countable chain unions. For example, is the lower Baer radical class closed under countable chain unions? It seems reasonable to ask whether or not every radical class of associative rings is closed under countable chain unions, or, in the special case of axiomatic radical classes, we have

Question 2. If $\mathscr{R}$ is an axiomatic radical class of associative rings, is $\mathscr{R}$ closed under countable chain unions, and hence definable by universal-existential axioms?

Another connection with model theory involves the closure of a radical class under homomorphic images (R1). An axiomatic model class is closed under homomorphic images if and only if it can be defined by positive sentences. A positive sentence is one which results from the quantification of a formula that can be built up using conjunction and disjunction but not negation. See [3, § VI. 5] and the references there, or [7, § 45].

The Jacobson radical for associative rings has an axiomatic semisimple class. Certain properties of the Jacobson radical are stated as abstract hypotheses for Proposition 2.

Proposition 2. Suppose that $\mathscr{R}$ is a radical class of associative rings defined by an axiom of the form $\forall x \exists y p(x, y)=0$, where $p(x, y)$ is a ring word formed using variables $x$ and $y$ and ring operations. Suppose further that the following three conditions hold:

(1) $\mathscr{R}$ is hereditary for left ideals. That is, if $A \in \mathscr{R}$ and 
$L$ is a left ideal of $A$, then $L \in \mathscr{R}$.

(2) $\mathscr{R}(A)$ contains every left ideal of $A$ that belongs to $\mathscr{R}$.

(3) If $a \in A$ and $A$ satisfies $\exists y p(a, y)=0$ and $A a=(0)$, then $Z . a \in \mathscr{R}$. Here $Z . a=\{n . a \mid n \in Z\}$ and $Z$ denotes the ring of integers.

Then $S(\mathscr{R})$ is also axiomatic, and $S(\mathscr{R})$ is defined by the following axiom:

$\sim \exists x[x \neq 0 \& \exists y p(x, y)=0 \& \forall z \exists y \exists u(p(z x, y)=0 \& y=u x)]$.

Proof. Let $\mathscr{S}$ denote the model class of associative rings having the long formal sentence as defining axiom. Suppose that $\mathscr{A} \in \mathscr{S}$, and let $a \in \mathscr{R}(A)$. Then $A$ satisfies $\exists y p(a, y)=0$, since $\mathscr{R}(A) \in \mathscr{R}$. But $A a$ is a left ideal of $\mathscr{R}(A)$, so $A a \in \mathscr{R}$ by condition (1). Thus $A$ satisfies $\forall z \exists y \exists u(p(z x, y)=0 \quad \& \quad y=u x)$. Since $A \in \mathscr{S}$, it must be the case that $a=0$. Hence $\mathscr{R}(A)=(0)$, so that $A \in S(\mathscr{R})$. Consequently, $\mathscr{S} \cong S(\mathscr{R})$.

Now suppose that $A \notin \mathscr{S}$. Then there exists some $a \in A, a \neq 0$, such that $A a \in \mathscr{R}$. By condition (2), $A a \leqq \mathscr{R}(A)$. If $A a \neq(0)$, then $\mathscr{R}(A) \neq(0)$, so $A \notin S(\mathscr{R})$. If $A a=(0)$, then $Z \cdot a \in \mathscr{R}$, by condition (3). But in this case $Z . a$ would be a left ideal of $A$ since $A(Z . a)=(0)$. Thus $Z . a \subseteq \mathscr{R}(A)$, by condition (2). Since $Z . a \neq(0)$, $\mathscr{R}(A) \neq(0)$, so again $A \notin S(\mathscr{R})$. Consequently, $S(\mathscr{R}) \subseteq \mathscr{S}$.

Question 3. For which other axiomatic radical classes do the hypotheses of Proposition 2 hold? More generally, what conditions on an axiomatic radical class suffice to imply that the corresponding semisimple class is also axiomatic?

Proposition 3. Suppose that $\mathscr{R}$ is a radical class of associative rings defined by an axiom of the form $\forall x \exists y p(x, y)=0$, where $p(x, y)$ is a ring word, and assume that $S(\mathscr{R})$ is axiomatic. Then, for every ultraproduct $A_{F}$ of associative rings $\mathscr{R}\left(A_{F}\right)=\mathscr{R}(A)_{F}$.

Proof. Suppose $A_{F}=\pi A_{i} / K$ is an ultraproduct. For each $i$, let $R_{i}=\mathscr{R}\left(A_{i}\right)$. Then $\mathscr{R}\left(A_{F}\right) \subseteq L / K$ by the Main Theorem. Take any $x \in L$. Then $H=\left\{i \mid x(i) \in R_{i}\right\} \in F$. If $i \in H$, then $x(i) \in R_{i}$ and so there exists $y_{i} \in R_{i}$ such that $p\left(x_{i}, y_{i}\right)=0$. Define $y \in L$ by $y(i)=y_{i}$ if $i \in H$, otherwise $y(i)=0$. But then $\{i \mid p(x, y)(i)=0\} \supseteqq H$ and therefore $p(x, y) \in K$. Thus $L / K \in \mathscr{R}$, and hence $L / K \cong \mathscr{R}\left(A_{F}\right)$. Consequently $\mathscr{R}\left(A_{F}\right)=L / K \cong \mathscr{R}(A)_{F}$.

Proposition 3 remains true provided $\mathscr{R}$ is defined by any collection of axioms of the form $\forall x_{1} \cdots \forall x_{m} \exists y_{1} \cdots \exists y_{n} p\left(x_{1}, \cdots, x_{m}, y_{1}, \cdots, y_{n}\right)=$ 0 , and the proof is similar. 
The Jacobson radical and the $V(P, N)$ radicals are equational classes, or varieties in the sense of universal algebra. The $V(P, N)$ radicals are subvarieties of associative rings. The Jacobson radical is not a subvariety of associative rings, but is an "extended" variety subordinate to the variety of associative rings. The Jacobson radical is an equational class over the operator domain which extends the ring operations with a quasi-inverse operation, and for which an axiom stating the existence of quasi-inverses of elements is added to the usual ring axioms. It is shown in [5] that not every hereditary radical class of associative rings that is closed under products is necessarily a variety. Both the Jacobson radical and the $V(P, N)$ radicals are closed under equalizers of ring homomorphisms. That is, if $f, g: R \rightarrow S$ are ring homomorphisms, where $R$ and $S$ belong to the radical class $\mathscr{R}$, then the equalizer subring $E=\{r \in R \mid f r=g r\}$ belongs to $\mathscr{R}$. It can be shown that if a radical class $\mathscr{R}$ of associative rings is closed under products and equalizers, then $\mathscr{R}$ is close under countable chain unions. Hence, an axiomatic radical class that is closed under equalizers can be defined by universal-existential axioms.

\section{REFERENCES}

1. S. A. Amitsur, Prime rings having polynomial identities with arbitrary coefficients, Proc. London Math. Soc., 17 (1967), 470-486.

2. T. Anderson, N. Divinsky, and A. Sulinski, Hereditary radicals in associative and alternative rings, Canad. J. Math., 17, No. 4 (1965), 594-603.

3. P. M. Cohn, Universal Algebra, Harper and Row, New York, 1965.

4. N. J. Divinsky, Rings and Radicals, University of Toronto Press. Toronto, 1965.

5. B. J. Gardner, Radical classes of regular rings with artinian primitive images, Tech. Report No. 136, Nov. 1979, The University of Tasmania, Hobart.

6. B. J. Gardner and P. N. Stewart, On semisimple radical classes, Bull. Austral. Math. Soc., 13 (1975), 349-353.

7. G. Gratzer, Universal Algebra, D. Van Nostrand, Princeton, 1968.

8. M. Gray, A Radical Approach to Algebra, Addison-Wesley, Mass., 1970.

9. I. N. Herstein, Noncommutative Rings, MAA Carus Monographs, No. 15, 1968.

10. A. E. Hoffman, Direct sum closure properties of radicals, J. Nat. Sci. Math. Lahore, 10 (1970), 53-58.

11. E. Posner, Prime rings satisfying a polynomial identity, Proc. Amer. Math. Soc.,

11. (1960), 180-184.

12. C. Roos, A class of regularities for rings, J. Austral. Math. Soc. (Series A), 27 (1979), 437-453.

13. R. Wiegandt, Radical and Semisimple Classes of Rings, Queens Papers in Pure and Applied Math. No. 37, Queen's University, Kingston, Ontario, 1974.

Received May 30, 1980 and in revised form March 9, 1981.

California State Polytechnic University

POMONA, CA 91768 



\section{PACIFIC JOURNAL OF MATHEMATICS}

\section{EDITORS}

DONALD BABBITT (Managing Editor)

University of California

Los Angeles, California 90024

Hugo RossI

University of Utah

Salt Lake City, UT 84112

C. C. MOORE and ANDREW OGG

University of California

Berkeley, CA 94720

\section{J. DugundJI}

Department of Mathematics University of Southern California Los Angeles, California 90007

R. Finn and J. Milgram Stanford University

Stanford, California 94305

\section{ASSOCIATE EDITORS}
R. ARENS
E. F. BeCKENBACH
B. H. Neumann
F. WoLF
K. YoSHIDA

\section{SUPPORTING INSTITUTIONS}

UNIVERSITY OF ARIZONA

UNIVERSITY OF BRITISH COLUMBIA

CALIFORNIA INSTITUTE OF TECHNOLOGY

UNIVERSITY OF CALIFORNIA

MONTANA STATE UNIVERSITY

UNIVERSITY OF NEVADA, RENO

NEW MEXICO STATE UNIVERSITY

OREGON STATE UNIVERSITY
UNIVERSITY OF OREGON

UNIVERSITY OF SOUTHERN CALIFORNIA

STANFORD UNIVERSITY

UNIVERSITY OF HAWAII

UNIVERSITY OF TOKYO

UNIVERSITY OF UTAH

WASHINGTON STATE UNIVERSITY

UNIVERSITY OF WASHINGTON 


\section{Pacific Journal of Mathematics}

\section{Vol. 97, No. $1 \quad$ January, 1981}

Charles A. Asmuth and Joe Repka, Tensor products for $S L_{2}(\mathfrak{k})$. II.

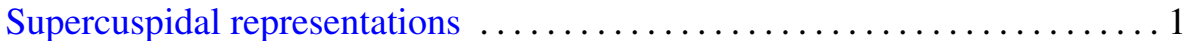

Joseph Barback, On finite sums of regressive isols . ................. 19

Matthew G. Brin and Daniel Russell McMillan, Jr., Generalized

three-manifolds with zero-dimensional nonmanifold set ............29

Kun Soo Chang, Converse measurability theorems for Yeh-Wiener space . . . 59

Christopher Brian Croke, A "maximal torus" type theorem for complete

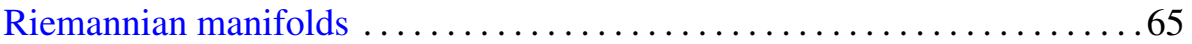

Gustave Adam Efroymson, Sums of squares in planar Nash rings . . . . . . 75

John Robert Fisher, Axiomatic radical and semisimple classes of rings . . . .81

Betty Kvarda, Consecutive integers for which $n^{2}+1$ is composite .......93

Roosevelt Gentry, New diagram proofs of the Hausdorff-Young theorem

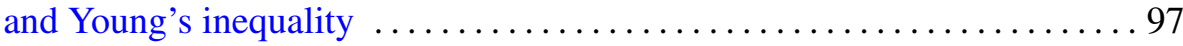

Patrick M. Gilmer, Topological proof of the $G$-signature theorem for $G$

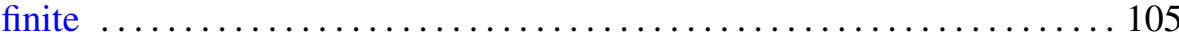

Chung Wei Ha, A noncompact minimax theorem .................. 115

James J. Hebda, Manifolds admitting taut hyperspheres ................ 119

Takayuki Kawada, Sample functions of Pólya processes ............. 125

Peter K. F. Kuhfittig, Common fixed points of nonexpansive mappings by

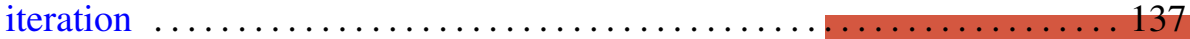

James Thomas Loats and Judith Roitman, Almost rigid Hopfian and dual

Hopfian atomic Boolean algebras .......................... 141

Roger McCann, On embedding semiflows into a radial flow on $l_{2} \ldots \ldots \ldots 151$

John McDonald, Closed orbits of convex sets of operators on the disk

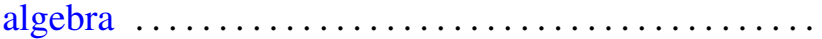

Mark D. Meyerson, Convexity and the table theorem .............. 167

Arnold William Miller, Generic Souslin sets . . . . . . . . . . . . . . . . 171

Takemi Mizokami, On the closed images of paracomplexes $\ldots \ldots \ldots \ldots \ldots 3$

Jagannadham Venkata Pakala and Thomas Stephen Shores, On

compactly packed rings $\ldots \ldots \ldots \ldots \ldots \ldots \ldots \ldots \ldots$

Andrew Pletch, Strong completeness in profinite groups

Wilbur Carrington Whitten, Inverting double knots

James Juei-Chin Yeh, Existence of strong solutions for stochastic

differential equations in the plane 